\title{
Biografía y memoria familiar
}

\section{Biography and Family Memoirs}

\author{
Laura Adriana Hernández Martínez \\ (D) orcid.org/0000-0001/7233-4518 \\ Universidad Autónoma Metropolitana-Iztapalapa, México \\ Departamento de Filosofía \\ lauraxanum@hotmail.com
}

Resumen: Este artículo aborda los dilemas que debe enfrentar un biógrafo que es familiar del personaje histórico biografiado. Se analiza este problema combinando el concepto de memoria colectiva de M. Halbwachs con la perspectiva dialógica de la biografía de M. Bajtín, en el afán de resolver así la tensión entre memoria familiar y discurso histórico. En virtud de que Paulino Martínez fue desaparecido cuando desempeñaba el cargo de jefe de la delegación zapatista a la Convención de Aguascalientes, este hecho traumático es estudiado desde la perspectiva de la postmemoria de M. Hirsch y el interés del arte contemporáneo en la creación de una nueva historiografía que permita sacar a la luz los discursos silenciados de los derrotados.

Palabras clave: biografía; postmemoria; zapatismo; memoria familiar; heterotopía.

Abstract: This article explores the dilemmas faced by biographers who are related to their historical subjects. This problem is analyzed by combining M. Halbwachs' concept of collective memory with M. Bajtín's dialogic perspective on biography and the goal of resolving the tension between family memoirs and historical discourse. Since Paulino Martínez went missing while serving as the Leader of the Zapatista Delegation at the Aguascalientes Convention, this traumatic event has been studied through M. Hirsch's post-memory perspective and contemporary art's interest in creating a new historiography that sheds light on the silenced discourse of the defeated. 
Key words: biography; post-memory; zapatismo; family memoir, heterotopia.

\begin{abstract}
Sólo tiene derecho a encender en el pasado la chispa de la esperanza aquel historiador traspasado por la idea de que ni siquiera los muertos estarán a salvo del enemigo, si este vence. Y este enemigo no ha dejado de vencer.
\end{abstract}

Walter Benjamin

\title{
PRESENTACIÓN
}

$\boldsymbol{E}^{\mathrm{s}}$ ste trabajo es, antes que nada, un intento por perfilar algunas de las pe-

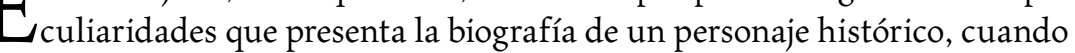
es narrada por uno de sus descendientes. De tal singularidad se desprenden dilemas éticos que debe resolver el autor y que condicionan la forma que tendrá el relato de esa vida que se pretende redondear, con la convicción de que merece ser contada. En este caso, el personaje es mi bisabuelo: Paulino Martínez, jefe de la delegación zapatista a la Convención de Aguascalientes que fue desaparecido en la ciudad de México la noche del 13 de diciembre de 1914, por lo que podemos considerarlo un mártir revolucionario. Sin embargo, la historia oficial de la revolución mexicana no lo ha incluido en el panteón de los héroes y apenas aparece mencionado como un periodista que fue precursor revolucionario y que pronunció un discurso beligerante en Aguascalientes contra Carranza, a quien comparó con Madero, como representantes de esa oligarquía que desde la caída de Díaz había impedido que se cumplieran los ideales plasmados en los planes revolucionarios de San Luis y de Ayala. Una afrenta al poder que Vasconcelos (1958) considera la causa de su asesinato en La tormenta (p. 137).

Mi familia siempre mantuvo un estrecho vínculo con José Vasconcelos, a quien consideraban uno de los pocos políticos que se había preocupado por su bienestar y el reconocimiento público de su participación en la revolución mexicana. Tanto fue así, que quien fuera su secretaria cuando dirigía la Biblioteca Nacional fue mi madrina. Y es que Vasconcelos (1958) regaló a mi bisabuelo palabras de enorme admiración, como las que dedicó a su discurso en la Convención de Aguascalientes:

\section{(이요 $\circledast$}


Don Paulino Martínez pronunció uno de los pocos discursos nobles, valientes, libres, que en la asamblea se dijeron. Arremetió contra el régimen militar que se infiltraba en la revolución... Entre todos los que en la Convención hablaron, nadie representó mejor los intereses de México que don Paulino Martínez y nadie puso atención a lo que dijo. Don Paulino, indio y ex obrero y veterano de las luchas contra la opresión capitalista del porfirismo y, además, periodista, no asesino, era el auténtico representante de la mayoría vejada. Los carranclano-pochistas, los que más tarde serían gobernadores y presidentes por la gracia yankee, escucharon a Don Paulino con displicencia... Don Paulino era un "pendejo" que se había enfrentado desde joven a Don Porfirio y se había pasado la vida escapando de la cárcel... ¡Qué pendejo Don Paulino!... Pos a poco cree que la revolución se hizo para que sigamos de pobres (pp. 118-119).

Como bisnieta de Paulino Martínez, tomé la decisión de escribir su biografía como un compromiso que contraía con mi familia de dar a conocer su vida y su terrible muerte, y que se originaba del profundo afecto que tuve hacia mi abuelo materno, Arturo, el hijo menor de Paulino Martínez y de Crescencia Garza. Él fue quien, en mi infancia, en uno de esos paseos inolvidables a su lado, me señaló visiblemente acongojado el lugar donde vivían cuando desapareció su padre, en el callejón de Leandro Valle, a un costado de la iglesia de Santo Domingo. No son ajenos a esa convicción de que había que denunciar el crimen, los largos relatos de mi abuela paterna, Aurora, hermana mayor de Arturo, quien me contó las grandes epopeyas revolucionarias, como la entrada de Madero a México, salpicadas de detalles y anécdotas de la situación familiar, siempre plagada de necesidades por la pobreza en la que siempre vivieron, amén de la implacable persecución de la policía a la que estuvieron sometidos sin tregua.

Es evidente, entonces, que me une a mi personaje un lazo afectivo, a pesar de no haberlo conocido, a través de sus hijos, mismo que pondría en duda la objetividad de mi relato, desde el punto de vista de la historia académica. Sin embargo, coincido con Ranciére en que si la historia, como disciplina científica, es un acto de escritura que consiste en configurar un régimen de verdad que se propone distanciarse de la literatura, existen otras formas de escritura de la historia que no pretenden superar esa condición literaria, sino que la consideran indispensable para otorgar un espacio de expresión a la memoria colectiva (véase Hernández, 2014a, pp. 289-298).

\section{(ㅇ)(1) $(3$}


Por otra parte, era indispensable llevar a cabo una investigación documental rigurosa para crear un nuevo archivo. La familia no contaba con nada, excepto un álbum de recortes que mi padre recuperó de la casa de su madre, donde mi abuela había reunido una serie de textos sin fechar, publicados en el periódico El Nacional por mi abuelo paterno, el periodista magonista Teodoro Hernández, ${ }^{1}$ donde se señalaba como autor material del crimen, a un tal Prócoro Dorantes que trabajaba por entonces bajo las órdenes de Vito Alessio Robles (jefe de la Policía de la ciudad) y que llegó a ser diputado del cuarto distrito electoral del Estado de México en 1918. ${ }^{2}$ También contaba con dos libros que hizo mi abuela Aurora, donde se encontraban muchos documentos y testimonios mimeografiados, que fueron sumamente útiles, a pesar de una mala edición, que se debió a que fueron hechos por cuenta propia.

Fue así que desde 2008 me di a la tarea de investigar la vida de mi bisabuelo en todos los archivos a mi alcance y conseguí reunir un buen número de cartas, panfletos y hasta conocí ejemplares de algunos periódicos de mi bisabuelo, recuperados por Carolina Villarroel, coordinadora del proyecto "Recovering the US Hispanic Literary Heritage" de la Universidad de Houston, quien por intermediación del muy estimado Antonio Saborit, me hizo llegar amablemente en microfilme. En esos años de intensa búsqueda apareció un documento en el Archivo CEHM Carso, que fue fundamental para mi futuro trabajo: la declaración que mi bisabuela hiciera siete años después de la desaparición de su marido, el 12 de abril de 1920, ante el quinto juez de lo penal, ${ }^{3}$ cuando Zapata ya había sido asesinado y Obregón estaba a punto de ser presidente, es decir, cuando el zapatismo había sido completamente derrotado. Junto a la declaración encontré también una carta de mi bisabuela, dirigida a Pablo González el 13 de mayo del mismo año, ${ }^{4}$ pidiéndole apoyo económico para poner una imprenta y poder salir de su difícil situación eco-

${ }^{1}$ Teodoro Hernández fue un periodista anarquista que colaboró en el periódico de Paulino Martínez: La Voz de Juárez, y que dirigió su propio periódico antiporfirista en Veracruz: La Voz de Lerdo. Estuvo encarcelado desde 1905 hasta la caída de Díaz y fue un activo magonista que aparece en la lista de los revisores del Plan Liberal. Durante muchos años, él y su esposa Aurora intentaron que Prócoro Dorantes y Vito Alessio-Robles fueran castigados por el crimen de Paulino Martínez, sin obtener ningún resultado.

${ }^{2}$ Legislatura XXVIII, Año I, Periodo Ordinario, Fecha 19180821. Número de Diario 3.

${ }^{3}$ LXVIII-1.21.2914.1. Archivo del Centro de Estudios de Historia de México Carso (Archivo CeHM Carso), México.

${ }^{4}$ LXVIII-1.21.2920.1. Archivo сенm Carso, México.

\section{(ㅇ)(1) $\$$}


nómica. Ahí anexaba la lista de las imprentas que le fueron decomisadas a su marido; un dato que fue fundamental para poder entender mejor los movimientos de mi bisabuelo y su familia en sus huidas, así como las reclusiones en la cárcel, que no sólo sufrió mi bisabuelo, ya que su esposa y dos de su hijas, Aurora y Clorinda, estuvieron recluidas en la cárcel de Belem en la época de Huerta y ya antes, Aurora con su madre en la Penitenciaría, en 1909.

Soy de la opinión de que este documento no fue relevante para ningún historiador porque podía considerarse como parte de un asunto meramente familiar, puesto que la muerte de Paulino Martínez había sido eliminada del relato oficial, amén de que Crescencia Martínez era mujer y la revolución, como toda guerra, ha sido abordado como un asunto de hombres. ${ }^{5}$

Es evidente que mi trabajo como biógrafa se asocia en este caso con dos tipos de memoria: la familiar y la que construyó la historia oficialista, las cuales entran en conflicto en tanto las versiones de los hechos no coinciden. Ambas forman parte de la memoria social, aunque la memoria familiar, a diferencia de la memoria histórica, sea una forma de la memoria colectiva. Es necesario, entonces, que dedique algún espacio a aclarar cómo entiendo esa diferencia entre la memoria familiar y el relato histórico, para lo cual me apoyaré en el trabajo realizado por Maurice Halbwachs (2004a) sobre la memoria colectiva. Con respecto a las formas de la escritura del pasado, retomaré la concepción dialógica y polifónica del lenguaje de Bajtín (1989), como una manera interesante de relacionar el carácter colectivo del relato con la forma enunciativa que el filólogo ruso postula para la biografía como género discursivo. Por otra parte, en esta perspectiva de la historia como un espacio de diálogo colectivo entre el pasado y el presente, es indispensable considerar al arte contemporáneo que se ha interesado por la memoria y el trabajo de archivo, en tanto permite la emergencia de discursos de resistencia que se producen en las intervenciones de los espacios urbanos, cuando se busca con ello poner a circular los relatos de aquellos que están ausentes del discurso histórico hegemónico. Para ello, me referiré al perfomance que yo misma realicé en Leandro Valle, número 20, el 13 de diciembre de 2014, exactamente 100 años después de la desaparición de mi bisabuelo, cuando en las calles se expresaba un enorme descontento civil por la desaparición de los 43 estudiantes de Ayotzinapa.

\footnotetext{
${ }^{5}$ Sobre este tema es destacable la novela de la historiadora y escritora Cristina Rivera Garza (1999).
}

\section{()(1) $(9$}




\section{MEMORIA FAMILIAR, MEMORIA SOCIAL E HISTORIA}

Lo que me permite cuestionar sucesos ya lejanos, y de los cuales no he sido testigo, proviene de una memoria familiar que mantiene a lo largo de los años el recuerdo de un acontecimiento traumático, como lo es la desaparición de un miembro de la familia. La historia también puede hacerlo, pero la diferencia estriba en que esta busca una imagen única y total de los acontecimientos que la inclina a interesarse: "sobre todo por las diferencias y se abstrae de los parecidos, sin los cuales no habría memoria, ya que sólo nos acordamos de los hechos cuyo rasgo común es que pertenecen a una misma conciencia" (Halbwachs, 2004a, pp. 85-86).

Por el contrario, la memoria colectiva es múltiple porque, en el caso de la memoria familiar, "debe abrazar en su campo ya no uno sino diversos grupos, en donde la importancia, el aspecto y también las relaciones mutuas cambian a cada momento" (Halbwachs, 2004b, p. 210). Esto se debe a que "el marco de la memoria familiar está constituido de nociones, nociones de personas y nociones de hechos, singulares e históricas en ese sentido, pero que tienen además todas las características de pensamientos comunes de todo un grupo, e incluso de varios" (Halbwachs, 2004b, p. 210).

La memoria individual no puede sustraerse, de acuerdo con Halbwachs, de la memoria colectiva, puesto que los recuerdos aparecen cuando son evocados por otros y constituyen puntos de referencia para la reconstrucción permanente del pasado desde el presente. En consecuencia, la memoria familiar tendrá recuerdos propios (lo que Halbwachs, 2004b, p. 201, denomina su "espíritu propio") que, aunque aluden a acontecimientos que han sido relevantes para esa familia, también se relacionan con recuerdos de otros colectivos sociales. De acuerdo con lo anterior, la desaparición de mi bisabuelo es un recuerdo de la memoria de mi familia pero también forma parte de la memoria colectiva del zapatismo y de aquellos que están interesados en escribir la historia de los derrotados de la revolución y señalar la vigencia de sus ideales. ${ }^{6}$

${ }^{6}$ El grupo de teatro campesino de Morelos marchó en diciembre de 2014, como lo hicieran Zapata y sus generales en diciembre de 1914 para entrar a la ciudad de México, al lado de Villa, después del encuentro que tuvieron en Xochimilco el 6 de diciembre, donde mi bisabuelo dio un hermoso discurso. Esta marcha, que no pudo entrar a la ciudad, por el control policiaco desplegado a partir de las protestas por la desaparición de los estudiantes de Ayotzinapa, fue recuperada por José Ramón Pedroza en el documental Los jinetes del tiempo,

\section{()(1) $\$$}


La condición colectiva de la memoria establece un diálogo entre la memoria individual del que recupera la historia de una vida con las memorias familiares y de grupos de otro tipo: políticos o artísticos, interesados en conseguir un relato polifónico que nos permita escuchar a los muertos olvidados por la historia.

\section{EL GÉNERO DISCURSIVO BIOGRÁFICO}

A pesar de que la biografía como género literario haya sido un tema discutido ampliamente por muchos pensadores destacados, creo que las notas de Bajtín sobre el tema son de enorme interés para abordar el problema que trato aquí, pues a diferencia de otras posiciones, más interesadas en un "pacto de verdad" entre el autor y el lector, Bajtín pone el acento en la importancia de la otredad en la biografía.

En toda biografía distinguimos al menos tres entidades que entran en juego: un autor, un narrador y un personaje. Sin embargo, biógrafo y biografiado son posiciones en el espacio discursivo del relato que se nos presentan como voces que se articulan en esa totalidad que constituye la narración, una vez que el narrador se funde como conciencia que interpreta la forma de la conciencia del otro:

ellos son dos, pero entre ambos no existe una oposición fundamental porque sus contextos valorativos son homogéneos, el portador de la unidad de la vida que es el héroe y el portador de la unidad de la forma que es el autor pertenecen a un mismo mundo valorativo [...] Ambos, el héroe y el autor, son otros y pertenecen a un mismo mundo autorizado y valorativo de otros. En la biografía no salimos fuera del mundo de los otros; tampoco la actividad creadora del autor nos hace rebasar estos confines: toda esta actividad está incluida en el ser de la otredad, es solidaria con el héroe en su pasividad ingenua [...] hay dos conciencias pero no dos posiciones valorativas, dos hombres, pero no un yo y otro, sino dos otros (Bajtín, 1989, p. 144).

estrenado en 2017. Se trata de un bello ejemplo de la memoria oral que han conservado, a lo largo de más de un siglo, los descendientes de aquellos zapatistas. Asimismo, es necesario hacer referencia al enorme trabajo que ha realizado el destacado historiador del zapatismo, Francisco Pineda, por aclarar la muerte de Paulino Martínez.

\section{(ㄷ)(1) $\$$}


De acuerdo con esto, es en el acto de la escritura biográfica -que es un acto consciente- cuando se consigue que la conciencia propia (lo que Bajtín llama el yo-para-mí) se encuentre con la conciencia del otro, puesto que la biografía, al igual que la autobiografía, son las formas donde podemos compartir los valores de la vida y el arte que "son formas y valores de la estética de la vida" (Bajtín, 1989, p. 134).

Estos valores están ligados con las acciones que conforman lo que Bajtín llama el momento heroico de la biografía, los cuales dan un sentido de gesta a la vida del héroe, quien si busca la gloria él mismo o su biógrafo busca dársela, de cualquier modo, es un deseo que no se puede deslindar del afán de reconocimiento por parte de los otros, en el marco de unos valores compartidos con ellos.

El segundo momento del valor biográfico es el amor, que Bajtín (1989) considera como "el deseo de ser amado, la comprensión, la visión y la constitución de una persona en una posible conciencia ajena y amorosa" (p. 138). Con lo cual podríamos concluir que si el valor heroico es, sobre todo, un reconocimiento social que te permite pertenecer a una comunidad, el amoroso es el del encuentro con el otro: el de la empatía. Finalmente, Bajtín (1989) habla del valor de la fabulación que consiste en aceptar positivamente la vida del héroe y así imaginar el relato de esa vida, darle forma (p. 139). Los tres valores se fusionan en el carácter de la biografía como donación: "me es donada por otros y para otros, pero la poseo ingenua y tranquilamente" (p. 147).

La otredad, desde esta perspectiva, nos conduce necesariamente hacia una polifonía de voces que participan en la forma que se le otorga a esa vida de acuerdo con los valores estéticos y éticos que constituyen el cronotopo del relato. Bajtín (1989) caracteriza este género discursivo (el de contar la vida de otros), como un género en el que los enunciados se encadenan en un diálogo colectivo que identifica esa vida con el sentido de la vida, por esa razón, si "La frontera entre el campo de visión y el entorno en la biografía es inestable y no tiene importancia decisiva, el momento de simpatía tiene una importancia máxima. Así es la biografía” (p. 147).

Varias cuestiones resultan relevantes de estas ideas para la manera en que entiendo las particularidades de la biografía que estoy elaborando. La primera tendría que ver con el hecho de que Bajtín otorga un lugar importante a las emociones que se derivan de la decisión de ser biógrafo de alguien y que no son sentimientos aislados de la circunstancia práctica de darle forma al relato, puesto que se trata de valores de carácter ético-estético que com-

\section{(ㅇ)(1) $\$$}


parten el personaje y el autor, pero que, además, hacen posible que puedan encontrarse dos conciencias que, a la manera de un discurso interno, pueden dialogar como voces en el relato y compartir esos valores. De ahí que la admiración y el amor sean la fuente de las corrientes que fluirán en la escritura fabulística.

$\mathrm{Y}$ es, en ese sentido, que puedo imaginar a mi bisabuelo como un hombre aguerrido, casi temerario, que admiró enormemente a su padre, que era un chinaco, juarista, liberal y anticlerical, don Buenaventura Martínez, a quien honró llamando a su primer periódico El Chinaco y a otro La Voz de Juárez. Como su padre muere siendo muy niño, su madre, doña Dolores González, sin medios económicos para mantenerlo, decide meterlo al Seminario de Celaya, ciudad donde había nacido un 22 de junio de 1860. Al morir su madre, en 1877, huye de ahí y llega caminando con unos arrieros a la ciudad de México, en donde consigue trabajo como organista de la catedral metropolitana. Ahí comenzará una vida de persecución y penas que logra su unidad en la incansable convicción de que había que destruir al gobierno y cambiarlo por otro que resolviera los graves problemas de hambre e injusticia del país. Es por eso que decidí que el carácter de gesta heroica se tradujera en una estructura de tres partes, denominadas: primera rebelión, segunda rebelión y tercera rebelión. Cada una de ellas representa un espacio de confrontación con el representante del poder en turno: Porfirio Díaz, Francisco I. Madero y Venustiano Carranza. Esta estructura rompe la sincronía de la historia oficial en la que estos tres personajes no aparecen relacionados como representantes de la oligarquía. La primera rebelión comienza poco tiempo después de que Paulino Martínez conoce a militares opositores a Díaz, como Mariano Escobedo y Trinidad García de la Cadena, y a periodistas lerdistas que lo introducen en ese oficio. Debido a que su padre había sido un chinaco, es evidente que su simpatía hacia ellos fue inmediata y decidió unirse a la lucha contra Díaz. Eso explica que en 1888 ya tenga su imprenta por el rumbo de la Alameda y salga a la luz El Chinaco. Ese mismo año se ve obligado a huir a Laredo, quizá a causa de un discurso que pronunciara en el quiosco morisco de la Alameda en contra de Porfirio Díaz en septiembre. Ya en Texas, región conocida como el "corredor antiporfirista" por la cantidad de periodistas exiliados, colabora en el periódico El Mundo que dirigía el doctor Ignacio Martínez, cabeza de la primera revuelta contra Díaz en Laredo, en la que participa activamente el recién llegado. Después de la muerte del doctor Martínez, el movimiento lo encabezará Catarino Garza y el grupo guerrillero se conocerá

\section{()(1) $\circledast$}


con el nombre de "Los pronunciados". En esa época conoce a Crescencia con quien se casa antes de ser arrestado y encerrado en la cárcel de San Antonio en 1895, cuando Catarino Garza huye a Costa Rica.

Mi bisabuela pertenecía a una familia mexicana de Laredo que había llegado desde Monterrey. Esa fue la razón de que mi bisabuelo hubiera intentado toda su vida organizar una colonia en Chihuahua, aprovechando la ley de terrenos baldíos que, ahora supongo, debió haber sido un propósito que tenía que ver con un deseo de mi bisabuela y su familia. ${ }^{7}$

Una vez liberado, los Martínez empiezan a buscar formas de sobrevivir bajo la vigilancia permanente de la policía fronteriza, dirigida por Bernardo Reyes - gobernador de Nuevo León- en combinación con los rangers. Cambian de ciudad frecuentemente y viven penosamente de hacer trabajos de impresión. En 1899 mi bisabuelo dirige en San Diego, Texas, una escuela llamada Colegio Mejicano, ${ }^{8}$ y poco después, a principios del siglo, Paulino Martínez ya forma parte del Círculo Liberal Ponciano Arriaga, donde conoce a los Magón y otros anarquistas con quienes traba amistad. Como relata Turner (1967, p. 178), apoya la huelga de Río Blanco, lo que le cuesta tortura y encarcelamiento en Orizaba. Se une al movimiento maderista y llega a ser secretario del Partido Antirreeleccionista, al lado de Filomeno Mata y de José Vasconcelos. A Zapata lo conoce en 1909, cuando acompaña al candidato maderista Patricio Leyva en su campaña por la gubernatura de Morelos, y este le pide que sea asesor de los campesinos de Anenecuilco. Madero lo comisionará para publicar en Laredo, Texas, El Monitor Democrático, así como comenzar a organizar la entrada a México desde la frontera texana, una vez que Madero llega hasta ahí, después de su encarcelamiento en San Luis Potosí. Paulino Martínez participará también en la elaboración del Plan de San Luis del que, como da testimonio Camilo Arriaga hijo, mi abuela Aurora dobló los pliegos para ponerlos dentro de los periódicos y así comenzar la sublevación (Martínez, 1964, p. 38).

${ }^{7}$ En dos textos se refiere mi bisabuelo a su pretensión de establecer una colonia de texanos mexicanos en México. El primero es una carta dirigida a Bernardo Reyes, con fecha del 9 de febrero de 1899. DLI-28.5481.1. Archivo CeHM Carso, México; y el segundo es su panfleto Causas de la revolución en México y cómo efectuar la paz, impreso por Hourcade, Crews \& Co. en La Habana el 6 de enero de 1914, incluido en mi compilación de textos, Hernández (2014b).

${ }^{8}$ Este dato lo obtuve a partir de las cartas que envió a Bernardo Reyes, las cuales estaban escritas en papel membretado de dicho colegio. DLI-28.5481.1. Archivo CEHM Carso, México.

\section{(ㅇ)(1) $\$$}


En la segunda rebelión se aborda la ruptura con Madero por haber traicionado el Plan de San Luis, al imponer a Pino Suárez como vicepresidente y no haber encaminado su gobierno a resolver los problemas campesinos y obreros. En esta etapa define su adscripción al zapatismo y se rompen definitivamente sus lazos de amistad con Ricardo Flores Magón, quien lo acusa de traidor en Regeneración. ${ }^{9}$

La tercera gesta es la más breve en el tiempo, pues se inicia con su exilio en La Habana, a causa del golpe de Victoriano Huerta, y termina con su desaparición, que ocurre poco tiempo después de que hubiera regresado a México, al llamado de Emiliano Zapata, quien lo nombra jefe de la delegación zapatista a la Convención de Aguascalientes en la Convención Zapatista de Cuernavaca en octubre de 1914.

La visión del personaje como un héroe puede traducirse en la exigencia de un reconocimiento que puede ser de glorificación o de denuncia. La diferencia estriba en que la gloria aísla al personaje de los demás, mientras que la denuncia implica a la sociedad en un suceso personal. Los mártires son honrados por el sacrificio que han hecho por todos, en cambio, los triunfadores sobresalen y se distinguen de los demás. Tengo la completa certeza de que lo que hago no tiene como meta el reconocimiento de las "hazañas" de Paulino Martínez, sino dar a conocer la injusticia de su muerte. Un asunto ético que debe resolverse en una forma estética, a través de una enunciación que tenga una arquitectura en la que sea visible ese hecho en la imagen completa de la historia con mayúscula. Se trata de entrar desde otra pregunta a la verdad de lo sucedido, una que incorpora nuevas voces en el relato, en lugar de meros personajes que son descritos. Una perspectiva que, sin duda, tiene que ver con el amor, esto es, con la manera de entender la lealtad afectiva y familiar en un sentido colectivo. Se busca que el narrador no se separe de lo narrado y que, en el uso del archivo, como apunta Cristina Rivera Garza (2013, p. 114), se rescaten, más que voces, autorías cuyo efecto sea la posibilidad de vivir la memoria, traer el pasado al presente y convertir la fabulación en una aventura llena de señales que van trazando una trayectoria de los sucesos diferente a la de los relatos hegemónicos, caracterizados por el monologuismo.

9 Agradezco profundamente a Jacinto Barrera las conversaciones que tuvimos sobre este pleito, así como que me encauzara a investigar la importancia de las colonias en esa época, pues Flores Magón acusa a Paulino Martínez de robar dinero a la gente con el pretexto de incorporarlos a su proyecto. Regeneración, abril de 1911, en línea http://archivorebelde.org/

\section{(ㅇ)(1) $\$$}


La memoria familiar constituye, entonces, el marco en el que se sostiene el relato, su autenticidad como verdad de un linaje, quizá hasta secreto de una familia derrotada que encarnó la truncada revolución. Sin embargo, deja de ser una historia privada cuando se transforma en un relato de todos, y esa memoria familiar se incorpora a los relatos en circulación sobre el pasado. Es muy interesante que Bajtín haya planteado que el encuentro de las conciencias sólo se pudiera dar en una forma estética y que esa misma idea sea la que emerja en la discusión contemporánea sobre la relación entre archivo y arte. A ese aspecto de la memoria dedicaré la última parte de este breve ensayo.

\section{MEMORIA EN RESISTENCIA Y ARTE URBANO}

El arte sirve para limpiar los ojos Karl Kraus

La desaparición de Paulino Martínez ocurre cuando la Convención de Aguascalientes se ha trasladado a la ciudad de México, como gobierno provisional, al mando de Eulalio Gutiérrez. En esos mismos días, se decidía la conformación del gobierno provisional y, como señala su esposa, Paulino Martínez había sido propuesto por Villa y Zapata para el cargo de secretario de Gobernación; ${ }^{10}$ un puesto que, dos días después de su desaparición, tomaría Lucio Blanco, quien era el candidato de Eulalio Gutiérrez y otros integrantes del gobierno provisional. La versión oficial de la desaparición de Paulino Martínez quedó plasmada en La tormenta de José Vasconcelos (1958), para quien el autor del crimen fue el implacable Rodolfo Fierro, apodado "El Carnicero":

Pensando que el primer paso de una organización democrática era el restablecimiento de las libertades municipales, Eulalio mandó un abogado de confianza y lo mandó expedir. A veces el acuerdo era lúgubre, como cuando nos llevaron la noticia de que la noche anterior había sido fusilado Paulino Martínez. Su viuda llegó a poco rato y acusaba al mismo Eulalio de complicidad; nadie sabía quién había ordenado la ejecución. Hasta que una mañana,

${ }^{10}$ Declaración de Crescencia Garza. LXVIII-1.21.2914.1. Archivo cenm Carso, México.

\section{(이요 $\$$}


Fierro en persona, confesó a Eulalio que él había hecho fusilar al ilustre viejo [...] por gusto [...] Más bien -pensé yo- porque no le perdonaban el discurso de Aguascalientes en que el veterano revolucionario condenó a los bribones que usaban la revolución para enriquecerse y asesinar (p. 137).

En su declaración, mi bisabuela relata cómo, durante toda la noche de la desaparición, fue enviada por diversas personas, cercanas al gobierno, a sitios diferentes donde se le aseguraba que estaba o había estado su marido. Uno de los lugares a los que se dirigió fue al hotel en que despachaba Eulalio Gutiérrez, donde le informaron que ya no estaba hospedado ahí, pero no supieron decirle a qué lugar se había trasladado. Ella no cuenta cómo lo supo al día siguiente, pero Vito Alessio-Robles (1979) sí relata ese traslado, sin mencionar la desaparición de mi bisabuelo, ni la fecha en que sucedió ese cambio de domicilio: "El general Gutiérrez, que vivía en el Hotel Palacio, se cambió a una suntuosa residencia en el primer tramo del Paseo de la Reforma, en la casa de uno de los Braniff, que a la llegada de Don Venustiano había sido ocupada por el general Rafael Buelna” (p. 403).

Páginas adelante dará su versión del crimen, en la que concluye que, a pesar de que se hicieron investigaciones, no se pudo saber nada sobre el responsable, ni sobre el paradero del cuerpo:

En la noche del 13 de diciembre, cuando don Paulino terminaba de cenar, se presentó en su casa un oficial. Le presentó una tarjeta del Gral. José Isabel Robles, ministro de la Guerra. Le rogaba que para tratar un asunto muy urgente se presentara inmediatamente en sus oficinas de la Secretaría a su cargo.

Martínez muy confiado acompañó al oficial. A la puerta los esperaba un automóvil con otros dos oficiales. Pero en vez de dirigirse a la Secretaría de Guerra lo condujeron, según pudo averiguarse después, al cuartel de San Cosme, en donde fue asesinado el viejo periodista. Eulalio Gutiérrez estaba furioso con asesinatos proditorios. Mandó practicar investigaciones. Nunca se supo dónde había sido sepultado el cadáver. José Isabel Robles negó haber enviado a esos oficiales y haberles dado una tarjeta suya. Muchos supieron que había sido sepultado en el interior de dicho cuartel (Alessio-Robles, 1979, p. 412).

Mi bisabuela relata, en cambio, que Alessio-Robles le dio la siguiente versión de los hechos:

\section{(이)(1) $(2$}


fui como a las seis de la mañana a ver al Sr. Eulalio Gutiérrez al "Hotel Palacio" donde me encontré con la sorpresa de que la misma noche que sacaron a mi esposo cambió su domicilio a la Calzada de la Reforma número 25 (casa de Braniff). Fui a su nuevo domicilio habiéndome recibido luego me dijo que regresara a las diez de la mañana que iba a mandar a llamar a José Isabel Robles para ver cómo estaba ese asunto.

A las diez de la mañana como me dijo el Sr. Gutiérrez me presenté acompañada de los Sres. Lic. Ángel Fernández de Córdova, Luis Rangel Viveros, Samuel Fernández, la Sra. Vda. De Martínez Carrión, y mi hijo Paulino Martínez. Estando allí vimos llegar a los Sres. José Isabel Robles, Manuel Chao, Vito Alessio Robles y otros.

Una vez llegados estos, a puerta cerrada comenzaron a deliberar sobre el asunto; de cuando en cuando abrían la puerta del cuarto donde se encontraban, nos veían y volvían a cerrar. A invitación del Sr. Ángel Fernández de Córdova nos fuimos a tomar una taza de café, la Sra. Vda. De Martínez Carrión y yo. Cuando nosotros ya nos encontrábamos allí, salieron todos de la junta y dijeron al señor Fernández que la noche del trece de diciembre habían matado a mi esposo a espaldas de la fábrica "El Progreso" y que ponían a la policía a nuestra disposición.

Dejé pasar el dolor que me había causado semejante noticia, y al tercer día me presenté ante el Sr. Inspector de Policía, Ing. Vito Alessio Robles; habiéndome preguntado que qué le informaba sobre el asunto de mi esposo, le contesté que a eso iba, a que me informara a lo que me contestó: "señora, a Don Paulino lo mataron en la fábrica 'El Progreso' a palos quemando después sus restos". 11

El encuentro de estos dos relatos fue lo que me dio la pauta para abordar el crimen del bisabuelo como la puesta en escena de las dos versiones de los hechos: una oficialista, que es la versión de Vasconcelos y de Vito Alessio-Robles y la de mi bisabuela, que se produjera 100 años después de la desaparición de mi bisabuelo, en el número 20 de ese callejón, donde había sido el convento de Santo Domingo y que, después de la Reforma, se convirtió en una vecindad que acabó sus días en el terremoto del 85 para ser entregada en comodato unos años después a Elba Esther Gordillo, con el fin de que se instalara ahí la Biblioteca del SNTE. La intervención en el sitio tomó la forma

${ }^{11}$ Declaración de Crescencia Garza. LXVIII-1.21.2914.1. Archivo cenm Carso, México.

\section{(ㅇ)(1) $\$$}


de un funeral sin cuerpo en memoria de Paulino Martínez, pero también en la de mi bisabuela, quien había cruzado tantas veces el umbral de la vecindad esa fatídica noche, y otras que siguieron hasta que se convenció de que no le sería entregado nunca el cuerpo de su marido. Eran los días en que el país se indignaba por la desaparición de los normalistas de Ayotzinapa y el secretario de Gobernación, Jesús Murillo Karam, presentaba la verdad histórica que coincidía con la respuesta que le diera Vito Alessio Robles (jefe de la policía) a Crescencia Garza cuando exigió la entrega del cuerpo de su marido: "para qué lo quiere, si lo mataron a palos y luego lo quemaron". Mi bisabuelo se convertía así, en el primer desaparecido posrevolucionario y con ello se trazaba un espacio histórico que abarcaba 100 años de impunidad y necropolítica.

La escritura de la historia de Paulino Martínez entraba en una crisis que me obligaba a abandonar la idea de un relato confinado al trabajo de archivo que sólo presentara los resultados documentales de la indagación. Era necesario conformar un nuevo archivo que confrontara esa historia oficial -la de Vasconcelos y Alessio Robles- que, además, daba diferentes versiones del crimen, con el relato de Crescencia. El performance constituiría, entonces, la creación de otro archivo, a partir de una ponencia que presenté sobre el crimen de mi bisabuelo, en el congreso que organizó la ENAH para celebrar el centenario de la Convención de Aguascalientes, ponencia que ya escribí con esa forma de careo, entre la versión oficial y lo que mi bisabuela había declarado en la policía. En la primera parte de la obra sólo había dos personajes: el asesino y Crescencia, que tenían soliloquios dialogados en los que se confrontaban dos maneras de ver lo mismo. La segunda parte era el funeral sin cuerpo, en donde yo traté de imaginarme el duelo de mi bisabuela como esa viuda desconsolada que ha perdido a su marido sin poder recuperar su cuerpo. Y aunque esto lo viví como una misión que había cumplido, y cuyo origen fue la imagen de mi abuelo acongojado en la plaza de Santo Domingo, lo que había hecho representaba un primer resultado de una tarea de investigación documental y teórica sobre la manera de narrar esa historia familiar que mostraba proyecciones inéditas sobre la historia de la revolución mexicana.

Sin embargo, un performance no es un relato escrito -aunque hubiera un texto-, es una acción que tiene el propósito de incidir en la memoria colectiva a partir de una intervención del espacio urbano - una apropiación del espacio- que abre la posibilidad de representar un acontecimiento que sucedió ahí y que nadie conoce. Esto es, se trataba de un intento por abrir una grieta en el discurso hegemónico, al intervenir un espacio de la ciudad Méxi-

\section{(이요 $\$$}


co que, como en gran parte del Centro Histórico, es un lugar donde duermen indigentes y ocurren asaltos por la noche.

Este nuevo uso del archivo en una intervención urbana, aunque sólo se centró en la desaparición, era la culminación de una larga batalla contra el poder que él y su esposa vivieron juntos y por la cual pasaron penurias inimaginables, fueron perseguidos sin tregua por la policía y encarcelados varias veces. Sin embargo, se mantuvieron leales uno al otro hasta el final, y a la muerte de mi bisabuelo, toda la familia se incorporó activamente a la lucha zapatista, en la clandestinidad. El crimen era también la culminación de todas las derrotas y, por eso, mi propuesta era la de producir un espacio de resistencia que diera lugar a una nueva forma de narrar la historia, en el convencimiento de que vivíamos en ese mismo "instante de peligro" que Benjamin (1977, p. 118) concebía como aquel en que estábamos obligados a la "recordación" del pasado, es decir, uno en que había que pensar el tiempo con el corazón para percibirlo y sentirlo como nuestro tiempo y así transformar nuestro presente.

Existen múltiples maneras en que se pueden producir estas nuevas narraciones que dan voz a los vencidos de la historia, pero quizá sea útil considerar que el trabajo que yo he realizado se sitúa dentro de la postmemoria. Un concepto acuñado por Marianne Hirsch (2012) para referirse a la memoria que es construida, no por los testigos vivenciales de un acontecimiento histórico, sino por los de la siguiente generación; en mi caso, dos generaciones después. Gibbons (2007, pp. 73-75) señala que, al igual que la contrahistoria y la contramemoria, la postmemoria es un tipo de memoria social que se distingue por recuperar lo que ha sido reprimido por aquellos que sufrieron la opresión y que fueron incapaces de expresar esa experiencia traumática. Una condición que le permite al narrador ser crítico y tener capacidad para buscar nuevas formas de contarlo. El artista sería entonces un segundo testigo y quien crea la postmemoria.

Por su parte, Ernst van Alphen (2009) es quien ha hablado del surgimiento de una nueva historiografía en el arte contemporáneo, que toma como modelo a la memoria, por su forma no lineal e imaginativa, en la que los recuerdos significativos pueden ser activados en el presente. Esa es la razón de que ese pasado se actualice de forma permanente en el presente de la memoria y sea tan afín al arte, puesto que "el arte no representa lo que ya sucedió, sino que establece las condiciones para relacionarse con lo sucedido" (Jill Benett, apud, Van Alphen, 2009, p. 46)

\section{(ㅇ)(1) $(9$}


No cabe duda de que el pensador que ha inspirado esta tendencia es Walter Benjamin (1977), quien, en sus famosas Tesis sobre la filosofía de la historia, dejó trazada la ruta estética para una nueva manera de escribir y hacer la historia, como una acción política cuyo tiempo está abierto y no discurre linealmente, sino mediante la libre disposición de las imágenes. Emerge de ahí la figura de un nuevo artífice de la historia, que es capaz de imaginar nuevas narrativas, a contracorriente de las dominantes, una actitud que se condensa en la noción del artista como historiador. Consideraré, entonces, esta figura del artista-historiador como un elemento clave para comprender cómo un espacio de memoria se convierte en un discurso de resistencia cuando un hecho histórico se enuncia bajo la forma de un juego de lenguaje inédito dentro del orden discursivo de la historiografía hegemónica. Es decir, cuando la historia se convierte en arte.

Esta revolución estética es una respuesta a lo que Joan Gibbons (2007) llama el "síndrome de la falsa memoria", que si bien, como concepto, tiene su origen en el psicoanálisis freudiano, se refiere aquí al engaño en que hemos vivido sobre nuestro pasado y que terminó cuando "las narrativas tradicionales patriarcales, imperialistas y colonialistas fueron transformadas e impugnadas en la segunda mitad del siglo xx por marginar grupos y sociedades" (p. 4). Dos ejemplos sobresalientes de este tipo de crítica son las obras de Edward Said y Michael Foucault que dieron paso a la necesaria vinculación de la historia con la cultura y que, añade Gibbons (2007): "se manifiesta claramente en el reclamo reciente por recuperar historias perdidas o marginadas en el arte contemporáneo" (p. 5).

Para el curador y teórico de arte francés, Nicolas Borriaud, el arte lleva la batuta en el derrumbe del historicismo como consecuencia de su capacidad para conectar niveles de realidad que están separados en el capitalismo, debido a la reificación de las relaciones humanas que siempre están mediadas por objetos. Una característica que Guy Debord (1967) atribuía a lo que él llamó la sociedad del espectáculo (en donde la representación de la realidad sustituye a la realidad) y que, en su opinión, también había derrotado al arte. Borriaud (2002) impugna a Debord, porque le parece que el papel del arte actual consiste, precisamente, en producir espacios donde se consiga el contacto entre las personas, aprovechando al máximo su condición esencialmente dialógica. A este nuevo arte lo denomina "relacional" (p. 14) y lo concibe como típicamente urbano; en virtud de que "El arte es el lugar que produce una sociabilidad específica que parte de los estados de encuentro que produ-

\section{()(1) $\circledast$}


ce la ciudad" (p. 16). El espacio urbano permite la aparición de un "intersticio social", en el sentido en que Marx usaba este concepto; esto es, como una grieta que se abre en el sistema capitalista cuando aparecen las relaciones que son posibles, más allá de aquellas que actúan de manera dominante.

Puesto que el arte está ligado a una forma, debiéramos considerar entonces, dice Borriaud (2002), que esa estructura de relaciones internas desborda su materialidad y se convierte en un principio relacionante, debido a que ahora "Producir una forma es inventar posibles encuentros; recibir una forma es crear las condiciones para un intercambio (como regresar un servicio en un juego de tenis)" (p. 23).

Este intersticio tiene una condición liminal, en el sentido que le daba a este término el antropólogo Víctor Turner (1988), debido a que la relación dialógica que destaca Borriaud en el arte contemporáneo sería imposible si las fronteras entre lo público y lo privado, así como entre lo recordado y lo olvidado, no se diluyeran para evitar la repetición y en su lugar dar paso a la rememoración reflexiva.

Por su parte, Gibbons considera que esta liminalidad debe verse a la luz de la filosofía de Henri Bergson (2007), para quien "el recuerdo representa el punto de intersección entre el espíritu y la materia" (p. 28); y el arte ha sido capaz de darle el locus indispensable para conseguir que la rememoración tenga un carácter comunitario, tal y como lo plantearon en su momento Pierre Nora y Marcel Proust (Gibbons, 2007, p. 6).

Desde esta perspectiva, la historia, la política y el arte formarían una unidad en el arte contemporáneo que, de acuerdo con Jacques Ranciére (1993), se asentaría en un fondo común que les otorga su cualidad de ser prácticas que producen ficciones y que, no por ello, deben ser mentiras; esto es: "Escribir la historia y escribir historias conciernen al mismo sistema de verdad" (p. 61). La relación de estas ficciones con el poder se da a través de lo que Ranciére llama el "régimen de lo sensible", que instituye la legitimación de lo que es visible y lo que es decible, es decir, de lo que se puede narrar para hacerlo visible. En este sentido, si el arte "hace visible lo que se piensa" es porque lo hace inteligible, a través de una forma que determina la manera en que hemos de sentir y percibir el mundo. Las formas son sistemas que ocurren en el espacio y en el tiempo y se apropian del espacio y el tiempo para determinar lo decible y lo visible y establecer los lugares que cada quien ocupa. Esto es lo que Ranciére entiende por política. Y en virtud de que la distribución de los lugares es precisamente la ficción y esta es el meollo de la historia

\section{()(1) $(9$}


(porque separa la ficción de la falsedad), podríamos hablar entonces de una racionalidad de la ficción que se expresa en una forma que hace inteligibles los fenómenos históricos. Siguiendo a Aristóteles, Ranciére (2014) considera que fingir no es engañar sino proponer estructuras inteligibles. Debido a que "lo real debe ser ficcionalizado para ser pensado" (p. 61).

La dimensión política de esta nueva estética produce una redistribución de los lenguajes y, como consecuencia, el borramiento de las fronteras entre las prácticas artísticas que, a su vez, redistribuyen las maneras de hacer y sus relaciones con las maneras de ser, con el único propósito de destruir la mentira de la normalidad. Por eso, dice Rancière (2014), "Las ficciones del arte y la política son [...] heterotopías [en el sentido de Foucault], más que utopías" (p. 64) que funcionan como contra-espacios: lugares ambiguos (intersticios) donde se puedan imaginar formas de vida diferentes, experiencias distintas a aquellas en que transcurre la vida cotidiana.

La intervención que yo realicé guarda semejanzas con otros trabajos que se llevaron a cabo para celebrar el centenario de la revolución mexicana. Uno que llamó especialmente mi atención fue la exposición: Espectografías. Memoria e historia, realizada en 2010 en el MUAC, en la que se buscaba sacar a la luz aquello que estaba oculto en las narraciones oficiales. En el texto de Ana María Martínez de la Escalera, incluido en el catálogo de la exposición, se aclara que una "memoria espectral" es aquella que se enfrenta a esa falsa memoria (podríamos decir ahora con Ranciére: esa ficción que sí es mentira) materializada en la conmemoración monumentalizada. La autora considera que esta memoria espectral es un discurso crítico, en el sentido foucaultiano, que se fundamenta en un saber de la gente que ha sido excluido por los saberes instituidos como poseedores de la verdad. Estos discursos, por su marginalidad y por defender solamente una verdad, suelen estar relacionados con pequeñas comunidades que se enfrentan a la verdad instituida. Su carácter crítico se deriva de que sacan a la luz esos discursos sedimentados por los archivos y permiten que se opongan a aquellos que han sido puestos en circulación para fijar y circular un sentido del pasado. Los discursos que surgen de estos saberes excluidos, en su opinión, deben considerarse como políticos porque, al manifestarse, son el germen de la polémica y la resistencia al poder, que es el modo en que pueden modificarse esas fuerzas y esas relaciones de poder.

El carácter polémico de los discursos en resistencia produce contrahistorias y contramemorias, pues cuando los documentos históricos de archi-

\section{()(1) $\circledast$}


vo se convierten en objetos estéticos y se sitúan en otros soportes y en otros órdenes, constituyen una contrahistoria que narra la historia oculta y sedimentada (la de los muertos y desaparecidos que no existen en la historia). La nueva historiografía sólo puede aparecer cuando es socializada y se convierte en un trabajo de memoria que se enfrenta a la memoria monumental de las instituciones. La contramemoria es, por ello, el fruto de una comunidad que rememora y no celebra: "Son actos de resistencia que se refieren a una memoria popular opuesta a una memoria institucionalizada o hegemónica" (Gibbons, 2007, p. 59).

La resistencia, sin embargo, no debe entenderse como un contrapoder, en virtud de que es una acción y no un resultado. El poder es el que reacciona ante las acciones de la resistencia que es propositiva y produce nuevas formas de vida posibles (intersticios). Esa es la diferencia entre una estética urbana, como acción política de resistencia, y las formas anteriores de lucha contra el poder, que sí eran contrapoderes puesto que, como señala atinadamente Judith Revel (2009), estaban condenados a generar otras formas de poder que a la larga podían ser formas incluso más autoritarias que las que habían destruido. La única alternativa ante esta situación, indica Revel, sería hacer más honda la "asimetría resistencial", ya que es ahí donde se sitúa la especificidad de lo político (p. 59) La diferencia entre el poder y la libertad está en que mientras esta es primigenia e inventiva, es una acción; el poder es genitivo, gestor y parasitario. Así podría concluirse con ella que "La apuesta de la resistencia deviene, por tanto, en cómo afianzar esa creatividad para ahondar la disimetría con el poder" (Revel, 2009, p. 60).

El discurso de resistencia es por tanto una acción política que tiene como eje la creación de espacios de memoria común que rompen la disposición de los lugares y los lenguajes hegemónicos para narrar el sentido del tiempo, como nuestro tiempo. Al poner el acento en la diferencia no se crea una nueva narración sino la posibilidad de que las diferentes historias ocupen un lugar común que funciona como un intersticio que, en su liminalidad, es el terreno propicio para que pueda germinar la libertad de ocupar cualquier sitio y no el que nos ha sido asignado por el poder. Es en ese lugar donde puedo encontrarme con mi bisabuelo en una unidad de conciencia que me revela mi historia con los otros, a través de su historia. Es probable que vaya por buen camino, aunque todavía queden muchos enigmas por resolver, pues qué otra cosa podría impulsarme a realizar esta tarea si no es este tan humano deseo de dialogar.

\section{(ㅇ)(1) $\$$}




\section{LISTA DE REFERENCIAS}

Alessio-Robles, V. (1979). La convención revolucionaria de Aguascalientes. México: INEHRM.

Alphen, E. van (2009). Hacia una nueva historiografía: Peter Forgacs y la estética de la temporalidad. Estudios Visuales, 6, 30-47.

Bajtín, M. (1989). Estética de la creación verbal. México: Siglo XXI Eds.

Benjamin, W. (1977). Tesis de filosofía de la historia. En Para una crítica de la violencia (pp. 110-141). México: Premià editora (Colección La Nave de los Locos).

Bergson, H. (2007). Materia y memoria. Ensayo sobre la relación del cuerpo con el espíritu. Buenos Aires: Cactus.

Borriaud, P. (2002). Relational aesthetics. Dijon: Les Presses du Reel.

Debord, G. (1967). La societè du spectacle. París: Buchet-Castel.

Gibbons, J. (2007). Contemporary art and memory. Images of recollection and remembrance. Londres: I. B. Tauris.

Halbwachs, M. (2004a). La memoria colectiva. Zaragoza: Prensas Universitarias de Zaragoza.

Halbwachs, M. (2004b). Los marcos sociales de la memoria. Barcelona: Anthropos.

Hernández, L. (2014a). Las escrituras de la historia en El hombre que amaba a los perros de Leonardo Padura. En J. Opatrný (coord.). El Caribe hispanoparlante en las obras de sus historiadores (pp. 289-298). Praga: Universidad Carolina de Praga/Editorial Karolinum

Hernández, L. (comp.) (2014b). Paulino Martínez. Ni pido ni doy cuartel. México: Consejo Editorial de la Cámara de Diputados.

Hirsch, M. (2012). The generation of postmemory. Writing and visual culture after the Holocaust. Nueva York: Columbia University Press.

Martínez, A. (1964). Antorchas de la revolución. México: s. e.

Martínez, A. (1966). Periodistas y periódicos ideológicos de la revolución mexicana (19001910). México: Prymer.

Martínez de la Escalera, A. (2010). Espectro y política de la memoria. En Espectografías: Memorias e historia. México: MuAc/unAm.

Rancière, J. (1993). Los nombres de la historia. Una poética del saber. Buenos Aires: Nueva Visión.

Rancière, J. (2014). El reparto de lo sensible. Estética y política. Buenos Aires: Prometeo Libros.

Revel, J. (2009). Sobre las resistencias, las subjetividades y lo común. En Multitud singular. El arte de resistir (pp. 56-66). Madrid: Museo Nacional Reina Sofía.

\section{()(1) $\$$}


Rivera Garza, C. (1999). Nadie me verá llorar. México: Tusquets.

Rivera Garza, C. (2013). Los muertos indóciles. Neoescrituras y desapropiación. México: Tusquets.

Turner, J. K. (1967). México bárbaro. México: Costa-Amic Editores.

Turner, V. (1988). The ritual process: Structure and anti-structure. Chicago: Alkine Publishing.

Vasconcelos, J. (1958). La tormenta. México: Jus.

\section{OTRAS FUENTES}

Archivo

CEHM CarsoArchivo del Centro de Estudios de Historia de México Carso, México.

\section{Bibliografía}

Martínez, P. (2014). Causas de la revolución en México y cómo efectuar la paz. En L. Hernández (comp.). Paulino Martínez. Ni pido ni doy cuartel (pp. 69-87). México: Consejo Editorial de la Cámara de Diputados. 